\title{
Transtorno do estresse pós-traumático em acidentes de trânsito: validação de escala
}

\author{
Marta Elisa Bringhenti ${ }^{1}$ - Universidade Federal de Santa Catarina, Florianópolis, Brasil \\ Caroline Di Bernardi Luft - Universidade Federal de Santa Catarina, Florianópolis, Brasil \\ Walter Ferreira de Oliveira - Universidade Federal de Santa Catarina, Florianópolis, Brasil
}

\begin{abstract}
Resumo
O objetivo do estudo foi realizar a validação da escala PCL-C para o rastreamento do transtorno do estresse pós-traumático entre vítimas de acidentes de trânsito. Participaram, 114 pessoas, que responderam à escala composta por 17 itens, divididos em três dimensões. Verificou-se a consistência interna por meio do coeficiente alfa de Cronbach com um valor de 0,94 . A análise fatorial exploratória, abrangendo a análise dos componentes principais e cargas fatoriais, com normalização Kaiser e rotação ortogonal pelo método oblimin, indicou que o construto da escala é unidimensional. Para adequação do ponto de corte realizou-se análise de sensibilidade e especificidade por meio da curva Receiver Operating Characteristic (ROC). O ponto de corte que obteve maior sensibilidade (1) foi 68 pontos com uma especificidade de 0,842 , mostrando-se o mais adequado para a discriminação do transtorno. A escala PCL-C apresentou qualidades psicométricas confiáveis para ser utilizada como um instrumento para o rastreamento do transtorno.

Palavras-chave: Transtorno do estresse pós-traumático, Acidentes de trânsito, Validação de testes, Análise fatorial, Confiabilidade.
\end{abstract}

\section{Post traumatic stress disorder checklist: validation for victims of traffic accidents}

\begin{abstract}
The study evaluates the validity of the PCL-C scale for screening victims of traffic accidents for post-traumatic stress disorder. One hundred and fourteen victims of traffic accidents participated in this study by completing the scale comprising 17 items, divided into three criteria related to the traumatic event. The Cronbach coefficient was used in order to verify the internal consistency with a value of 0.94 . The exploratory factor analysis was carried out to verify the construct validity, including the analysis of the main components and factor loadings with Kaiser normalization and orthogonal rotation by the oblimin method. The results of exploratory factor analysis indicate that de construct is one-dimensional. For the suitability of the cutoff point, sensitivity and specificity analysis was carried out through the Receiver Operating Characteristic (ROC) curve. The cutoff point which obtained the greatest sensitivity (1) was 68 points with specificity of 0.842 , this being the most suitable value for the identification of the disorder. The scale for the screening of post-traumatic stress disorder showed reliable psychometric qualities.

Keywords: Post-traumatic stress disorder, Traffic accidents, Validation of tests, Factor analysis, Reliability.
\end{abstract}

O transtorno do estresse pós-traumático (TEPT) é uma condição psicofisiológica que pode ocorrer após a vivência de eventos altamente estressantes. O indivíduo apresenta dificuldades de elaboração cognitivo-emocional e alterações psicofisiológicas em virtude das reações do organismo às agressões sofridas (Bremner, 2004; Graeff, 2003). As vítimas com TEPT sentem dificuldades para se desvincular das cenas e dos fatos e evidenciam estado de tensão e apreensão constantes com prejuízos elevados para a saúde e o bem-estar (American Psychiatric Association, 1995; Bremner, 2004; Organização Mundial da Saúde, 1993).

Os acidentes de trânsito encontram-se entre os eventos cuja magnitude da violência pode levar as vítimas a desenvolverem o TEPT (Norris, 1992). Essa magnitude está relacionada às limitações que o acidente impõe às vítimas como: sequelas físicas, risco de morte, prejuízos à saúde, afastamento do trabalho,

\footnotetext{
${ }^{1}$ Endereço para correspondência:

Rua Francisco Goulart, n. 96, apto. 101 - Bairro Trindade CEP 88036600 - Florianópolis-SC

E-mail: martabringhenti@yahoo.com.br
}

prejuízos econômico-financeiros, processo judicial, dependência de cuidadores, incapacitação temporária ou permanente. No Brasil, as consequências dos acidentes de trânsito ainda são pouco conhecidas e estudadas (Instituto de Pesquisa Econômica e Aplicada \& Associação Nacional dos Transportes, 2003; Vasconcellos, 2005), inclusive o próprio desenvolvimento do TEPT. Figueira e Mendlowicz (2003) afirmam que o transtorno tem recebido pouca atenção, tendo em vista o aumento da violência urbana e dos acidentes.

No Brasil há elevado número de acidentes de trânsito (Ministério da Saúde, 2008), com sérios prejuízos à saúde das vítimas por causa da extensão das lesões e da necessidade de tratamentos que envolvem longos períodos de tempo para a recuperação e a reabilitação. Estima-se que há elevado número de pessoas vivendo em condições de morbidade decorrente dos acidentes de trânsito (Instituto de Pesquisa Econômica e Aplicada, 2007). A dificuldade de se identificar e acompanhar as consequências dos acidentes de trânsito também se estende à dificuldade de identificar a manifestação do TEPT entre as vítimas. 
A aplicação de instrumentos devidamente validados para o rastreamento do TEPT possibilitaria a identificação precoce do transtorno e facilitaria o encaminhamento para profissionais habilitados a realizar o tratamento e o acompanhamento. A escala PCL-C foi desenvolvida para mensurar a magnitude do trauma ou se ele atinge determinado grau para que seja considerado um transtorno (Weathers, Litz, Herman, Huska \& Keane, 1993). Conhecido como PTSD Checklist - Civilian Version (PCL-C), a PCL-C foi desenvolvida por Weathers e cols. (1993). Possui duas versões: uma para avaliação de consequências de experiências militares e uma para avaliação das consequências de traumas diversos na população civil. Ambas as versões têm como base os critérios diagnósticos do DSM-III-R (American Psychiatric Association, 1987) para o TEPT. A validação proposta neste artigo se refere à versão civil.

A PCL-C é uma escala geral que pode ser usada em diversos grupos etários, desde adolescentes até idosos. Também pode ser utilizada para eventos traumáticos diversos, pois não contém questões específicas ao contexto do trauma. É um instrumento de rápida aplicação, aproximadamente 10 minutos, prático e generalizável (Weathers \& cols., 1993). Em diferentes tipos de estudos, a PCL-C tem apresentado características psicométricas satisfatórias no que diz respeito à validade e à confiabilidade (Andrykowski, Cordova, Studts \& Miller, 1998; Blanchard, JonesAlexander, Buckley \& Forneris, 1996; Smith, Redd, DuHamel, Vickberg \& Ricketts, 1999; Weathers \& cols., 1993). A escala PCL-C foi escolhida entre outras (Roso, 1998) pelo fato de haver estudos de validade semântica realizados no Brasil (Berger, Mendlowicz, Souza \& Figueira, 2004)

Reconhecendo-se que o TEPT afeta as pessoas em nível epidemiológico (Yehuda, 2002); considerando a importância de se identificar precocemente a manifestação do transtorno; a necessidade de instrumentos validados em português e que os acidentes de trânsito são eventos de elevada ocorrência com potencialidade para $O$ desencadeamento do TEPT (Kessler, Sonnega, Bromet, Hughes \& Nelson, 1995), o presente estudo tem o objetivo de realizar a validação da PCL-C entre vítimas de acidentes de trânsito.

\section{Método}

O estudo foi desenvolvido em duas etapas: a primeira abrangeu a análise da validade semântica da PCL-C - tradução realizada por Berger e cols. (2004) com sujeitos que sofreram acidentes de trânsito; e a segunda, a análise da validade de construto, de critério e de confiabilidade da escala, adaptada a partir dos resultados da primeira etapa.

\section{Participantes}

Participaram do estudo vítimas de acidentes de trânsito com no mínimo um mês de ocorrência do evento, período estimado para a caracterização do TEPT crônico (American Psychiatric Association, 1995). Foram excluídos da participação vítimas com traumas na região buçomaxiliar, em razão da dificuldade de comunicação, e menores de 16 anos.

\section{Primeira etapa}

Nesta etapa a PCL-C foi aplicada em 11 sujeitos, 7 homens e 4 mulheres com média de idade de 36,4 (DP=13,25). Foram feitas alterações da escala PCL-C (Berger \& cols., 2004) por algumas dificuldades identificadas entre os respondentes no início da pesquisa. A escala com as modificações feitas no presente estudo encontra-se no Anexo 1.

\section{Alteracões realizadas na escala PCL-C}

a. Antes do início da leitura dos itens destacou-se a consigna neste último mês... já presente nas instruções da escala traduzida por Berger e Mendlowicz (2004). Visou-se dar maior visibilidade à instrução inicial para que o leitor perceba que os itens se referem a aspectos do trauma revividos recentemente. Nos dois itens seguintes (item 1 e 2), foi incluída a consigna "Você tem tido...". As alterações serviram para guiar o leitor quanto ao que ele sente ou percebe no presente, relativo à sua reexperiência do trauma sofrido num passado relativamente distante, mas cujas lembranças são revividas com certa frequência. Observou-se que o leitor deixou de fazer perguntas como: "essa pergunta é em relação ao que eu sinto?", "você quer saber se eu tenho isso?".

b. O item 12 "Sentir como se você não tivesse expectativas para o futuro" foi alterado para: "Sentir-se sem expectativas para o futuro". Observou-se a dificuldade do leitor decidir se ele tinha ou não o sintoma, pela dificuldade de associar o sentido negativo (não) à experiência dele. Foram observadas dúvidas como: "não, eu não sinto isso, então tenho que responder o quê?" ou "sim, eu tenho isso, mas aqui diz que não, então tenho que colocar mais ou menos?".

c. Optou-se por transformar a escala que variava inicialmente de 1 (nada) a 5 (muito) para uma escala de 0 a 10 (0 - nada a 10 - totalmente). Muitos participantes relataram dificuldades em se posicionar na escala de cinco pontos, pois para eles não era fácil distinguir intensidades como "bastante", que valia 4 pontos, com "muito" que valia 5 pontos. Mesmo 
solicitando para que eles dessem uma nota de 1 a 5 , alguns participantes reclamaram "não é cinco, é mais...".

Nas escalas do tipo Likert, cada item possui cinco categorias de respostas que vão do "discordo totalmente" ao "concordo totalmente" (Oliveira, 2001). Já a escala do tipo Stapel é uma escala unipolar de 10 pontos destinada a mensurar tanto a direção quanto a intensidade das atitudes, de forma que a PCLC combina as vantagens da escala Likert com a Stapel. Tanto a escala Likert como a Stapel são recomendadas para medidas de atitudes e percepções (Oliveira, 2001), e a sua combinação deve levar em conta a complexidade e os objetivos da utilização do instrumento.

As alterações propostas se referem ao formato, consigna e escala. O conteúdo dos itens foi mantido de acordo com a escala traduzida para o português por Berger e cols. (2004).

\section{Segunda etapa}

$\mathrm{Na}$ segunda etapa foram considerados 103 participantes, sendo $13,6 \%$ mulheres $(\mathrm{n}=14)$ e $86,4 \%$ homens $(\mathrm{n}=86)$. A idade variou de 16 a 64 anos, com idade média de 30,8 anos (DP=10,48). Quanto ao estado civil, 61 indivíduos (59,24\%) eram solteiros, 37 casados $(35,9 \%)$ e cinco indicaram outra condição civil $(4,8 \%)$. Quanto à escolaridade, $10(9,8 \%)$ possuíam curso superior, $40(38,8 \%)$ tinham o ensino médio e 53 $(51,5 \%)$, ensino fundamental. Quanto à renda mensal bruta, $16(15,6 \%)$ referiram não possuir renda ou ter renda de até um salário mínimo, $69(67,0 \%)$, de um a dois salários mínimos e meio, $16(15,5 \%)$, de dois salários mínimos e meio a sete salários mínimos e apenas $2(1,9 \%)$ acima de sete salários mínimos.

\section{Instrumentos}

Aos participantes que aceitaram fazer parte do estudo foi apresentado o Termo Livre e Informado para leitura e assinatura. Após, os entrevistados foram convidados a preencher um questionário que continha questões sobre dados sociodemográficos, do acidente de trânsito e de saúde, que incluiu, por exemplo, a história de doenças psicossomáticas anteriores e posteriores ao acidente de trânsito, uso de medicação controlada e histórico de traumas psíquicos. Por último, os participantes receberam a escala PCL-C para ser respondida.

\section{Características da escala PCL-C}

A PCL-C é composta por 17 itens, divididos em três dimensões, cada uma delas com questões específicas baseadas nos critérios diagnósticos do
DSM-III-R e IV (American Psychiatric Association, 1987, 1995). As três dimensões da escala são:

- Reexperiência do trauma, que corresponde ao critério B do DSM IV e inclui os itens 1, 2, 3, 4 e 5, totalizando cinco itens.

- $\quad$ Evitação, que corresponde ao critério C do DSM IV e inclui os itens 6, 7, 8, 9, 10, 11 e 12, totalizando sete itens.

- Hiperestimulação, que corresponde ao critério D do DSM IV e inclui os itens 13, 14, 15, 16 e 17 , totalizando cinco itens.

A dimensão reexperiência do trauma inclui sinais e sintomas como revivescências, alterações do sono, pesadelos, sofrimento psicológico e reatividade fisiológica decorrente das lembranças do evento. A dimensão evitação inclui sinais e sintomas, como esforço para evitar pensamentos negativos, lugares e atividades que evoquem o evento, lapsos de memória, desmotivação e sentimento de futuro abreviado. A dimensão hiperestimulação inclui sinais e sintomas, como insônia, hipervigilância, sobressaltos e irritabilidade intensa (American Psychiatric Association, 1995; Weathers \& cols., 1993).

Os critérios A, E e F indicados no DSM IV (American Psychiatric Association, 1995) se referem à exposição ao evento traumático, à duração da perturbação que deve ser superior a um mês e aos prejuízos que o indivíduo sofre, decorrentes do transtorno, respectivamente.

A escala pode ser autoaplicada e o examinado indica a intensidade dos sintomas relativos à experiência do trauma. A análise da pontuação pode ser feita de duas formas: uma, pelo método da soma total de pontos, sugerida por Weathers e cols. (1993). Para que o diagnóstico do TEPT seja positivo é necessária a soma de 50 pontos ou mais. Cada resposta pode variar de 1 a 5 (Weathers \& cols., 1993). A outra forma é pelo número de itens em cada dimensão, conforme o DSM IV (1995). O indivíduo deve assinalar no mínimo um item da dimensão reexperiência, no mínimo três itens da dimensão evitação e no mínimo dois itens da dimensão hiperestimulação. Neste estudo o rastreamento do TEPT foi feito pelo número de itens, conforme indicação do DSM IV (1995), ou seja, foi observado se os participantes responderam no mínimo a um item da dimensão reexperiência, três itens da dimensão evitação e dois itens da dimensão hiperestimulação. A escolha do método estabelecido no DSM IV (American Psychiatric Association, 1995) se deve ao fato de que a intensidade dos itens foi alterada de 1 a 5 para 0 a 10 pontos e um novo ponto de corte foi sugerido. 


\section{Procedimentos}

Os dados foram coletados do início de novembro de 2007 ao final de fevereiro de 2008, no ambulatório de Traumatologia e Ortopedia do Hospital Regional São José (HRSJ), na cidade de São José e no Centro Catarinense de Reabilitação (CCR), na cidade de Florianópolis, ambos os municípios do estado de Santa Catarina. São instituições que fazem parte da rede púbica de saúde, estão vinculados a Secretaria da Saúde do Estado de Santa Catarina e atendem, entre outros, vítimas de acidentes de trânsito nos níveis de atenção de média e alta complexidade.

O presente estudo foi aprovado pelo Comitê de Ética e Pesquisa da Secretaria da Saúde do Estado de Santa Catarina, sob o número 0011.1907-07.

\section{Análise estatística}

Primeiramente, os dados foram inspecionados visualmente e por meio de gráficos de caixa e histogramas, visando identificar erros e valores desviantes. Procedeu-se a análise descritiva dos resultados, utilizando medidas de tendência central e variabilidade, incluindo análise de distribuição dos dados (histogramas e teste de Kolmogorov-Smirnov).

Quanto à validade de construto, essa foi verificada por meio da análise fatorial exploratória com normalização kaiser e rotação oblíqua (direct oblimin). Foi calculado o índice de Kaiser-Meyer-Olkin (KMO) para verificar a proporção da variância dos dados que pode ser considerada comum a todas as variáveis, ou seja, que pode ser atribuída a um fator comum. Adicionalmente, realizou-se o teste de esfericidade de Bartlett, que testa se a matriz de correlação é uma matriz identidade, o que indicaria que não há correlação entre as variáveis. Ambos os testes (KMO e Bartlett) indicam a adequação dos dados para a realização da análise fatorial.

Em primeiro lugar, realizou-se a análise dos componentes principais, para identificar quantas dimensões latentes a escala apresenta e a variação percentual que cada dimensão consegue explicar (com base nos eigenvalues). Observaram-se os valores das comunalidades e as cargas fatoriais nas respectivas dimensões sem rodar a matriz. Após, procedeu-se à rotação oblíqua, por meio do método oblimin. Utilizou-se esse método ortogonal porque as dimensões encontradas na escala estão correlacionados $(0,659)$. Em seguida procedeu-se à análise da confiabilidade da PCL-C avaliada pela consistência interna, verificada por meio do coeficiente alfa de Cronbach.

Para selecionar os pontos de corte mais adequados para a discriminação do TEPT, realizou-se a análise da curva Receiver Operating Characteristic
(ROC). A curva ROC é um procedimento que visa estabelecer os pontos de corte da forma mais adequada, calcular os índices de verdadeiro-positivo (sensibilidade) e de verdadeiro-negativo (especificidade) para todos os pontos de corte, relacionar esses dois indicadores em um gráfico bidimensional, obtendo-se, assim, a curva ROC (Norman \& Streiner, 2000). A área que se encontra sob a curva ROC é um índice global da precisão de discriminação que a escala oferece.

Assim, a curva ROC, no presente estudo, foi utilizada como um procedimento estatístico para readequar os pontos de corte sugeridos pelos autores da escala, uma vez que no decorrer da primeira etapa a intensidade dos itens foi alterada de 1 a 5 para 0 a 10 . Utilizou-se pressuposição de dados sem distribuição normal (não-paramétricos). A área sob a curva foi utilizada como um indicador da adequação dos pontos de corte, pois um escore sem capacidade de discriminar casos de "não-casos" tem uma área menor, abaixo de 0,5 , enquanto se esse indicador for próximo de 1, melhor habilidade discriminativa tem $\mathrm{O}$ instrumento. Quanto mais próximo o ponto estiver do canto superior esquerdo, menor taxa de erro.

Assim, a análise da curva ROC foi realizada com o objetivo de readequar os pontos de corte sugeridos pelos autores da escala, que neste estudo devem ser utilizados com o objetivo de rastrear o TEPT para obter evidências sobre a presença do transtorno. A utilização como um instrumento diagnóstico fica limitada uma vez que não foram utilizadas as técnicas diagnósticas tradicionais, consideradas como padrão-ouro. Ao obter evidências sobre a presença do TEPT, com escores acima de 68 pontos, os prováveis casos do transtorno devem ser encaminhados para atendimento clínico e confirmação do diagnóstico.

Originalmente o ponto de corte sugerido por Weathers e cols. (1993) é 50 pontos, com uma variação de intensidade da escala entre 1 e 5. Para a confirmação diagnóstica entre casos e não-casos os autores utilizaram a Entrevista Clínica Estruturada, módulo para o TEPT, do DSM-III-R. Foi encontrada uma sensibilidade de 0,82, uma especificidade de 0,83 e um índice kappa de 0,64 (Weathers \& cols., 1993).

\section{Resultados}

A amostra de dados foi considerada adequada para a análise fatorial da escala PCL-C, pois o índice Kaiser-Meyer-Olkin encontrado foi de 0,916 , que pode ser considerado um valor próximo de excelente $(0=$ inadequada; 1 =excelente). $O$ teste de esfericidade de Bartlett indicou que os dados são apropriados para 
análise fatorial $\left(\mathrm{X}^{2}=1326,84, \mathrm{p}<0,001\right)$. Assim, foram realização da análise fatorial.

assegurados os pré-requisitos necessários para a

Tabela 1 - Cargas fatoriais dos itens e dimensões extraídos da PCL-C

\begin{tabular}{|c|c|c|c|c|c|}
\hline & & \multicolumn{2}{|c|}{ Não rodada } & \multicolumn{2}{|c|}{ Oblimin (pattern) } \\
\hline Item & $\mathrm{H}^{2}$ & Dimensão 1 & Dimensão 2 & Dimensão 1 & Dimensão 2 \\
\hline 15. Ter dificuldades para se concentrar & 0,782 & 0,790 & $*$ & 0,959 & $*$ \\
\hline $\begin{array}{l}\text { 13. Ter problemas para pegar no sono ou } \\
\text { para continuar dormindo }\end{array}$ & 0,712 & 0,759 & * & 0,908 & $*$ \\
\hline $\begin{array}{l}\text { 14. Sentir-se irritável ou ter explosões de } \\
\text { raiva }\end{array}$ & 0,736 & 0,817 & * & 0,832 & * \\
\hline $\begin{array}{l}\text { 10. Sentir-se distante ou afastado das outras } \\
\text { pessoas }\end{array}$ & 0,686 & 0,783 & * & 0,819 & * \\
\hline $\begin{array}{l}\text { 16. Estar "superalerta", vigilante ou "em } \\
\text { guarda" }\end{array}$ & 0,729 & 0,839 & * & 0,738 & * \\
\hline $\begin{array}{l}\text { 9. Perda de interesse nas atividades de que } \\
\text { você antes costumava gostar }\end{array}$ & 0,634 & 0,759 & * & 0,771 & * \\
\hline $\begin{array}{l}\text { 12. Sentir como se você não tivesse } \\
\text { expectativas para o futuro }\end{array}$ & 0,592 & 0,717 & $*$ & 0,784 & $*$ \\
\hline $\begin{array}{l}\text { 17. Sentir-se tenso ou facilmente } \\
\text { sobressaltado }\end{array}$ & 0,712 & 0,836 & * & 0,685 & $*$ \\
\hline $\begin{array}{l}\text { 11. Sentir-se emocionalmente entorpecido ou } \\
\text { incapaz e ter sentimentos amorosos pelas } \\
\text { pessoas que lhe são próximas }\end{array}$ & 0,536 & 0,705 & * & 0,688 & * \\
\hline $\begin{array}{l}\text { 8. Dificuldades para lembrar-se de partes } \\
\text { importantes de uma experiência estressante } \\
\text { do passado }\end{array}$ & 0,386 & 0,619 & * & 0,434 & * \\
\hline $\begin{array}{l}\text { 5. Sentir sintomas físicos (por exemplo, } \\
\text { coração batendo forte, dificuldade de } \\
\text { respirar, suores) quando alguma coisa lembra } \\
\text { você de uma experiência estressante do } \\
\text { passado? }\end{array}$ & 0,737 & 0,632 & 0,581 & * & 0,966 \\
\hline $\begin{array}{l}\text { 1. Memória, pensamentos e imagens } \\
\text { repetitivas e perturbadores referentes a uma } \\
\text { experiência estressante do passado? }\end{array}$ & 0,699 & 0,711 & 0,440 & * & 0,832 \\
\hline $\begin{array}{l}\text { 4. Sentir-se muito chateado ou preocupado } \\
\text { quando alguma coisa lembra você de uma } \\
\text { experiência estressante do passado? }\end{array}$ & 0,718 & 0,746 & 0,402 & $*$ & 0,802 \\
\hline $\begin{array}{l}\text { 3. De repente, agir ou sentir como se uma } \\
\text { experiência estressante do passado estivesse } \\
\text { acontecendo de novo (como se você a } \\
\text { estivesse revivendo)? }\end{array}$ & 0,726 & 0,768 & 0,369 & * & 0,772 \\
\hline $\begin{array}{l}\text { 2. Sonhos repetitivos e perturbadores } \\
\text { referentes a uma experiência estressante do } \\
\text { passado? }\end{array}$ & 0,625 & 0,709 & 0,348 & $*$ & 0,722 \\
\hline $\begin{array}{l}\text { 7. Evitar atividades ou situações porque elas } \\
\text { lembram uma experiência estressante do } \\
\text { passado? }\end{array}$ & 0,602 & 0,746 & 0,402 & * & 0,620 \\
\hline $\begin{array}{l}\text { 6. Evitar pensar ou falar sobre uma } \\
\text { experiência estressante do passado ou evitar } \\
\text { ter sentimentos relacionados a esta } \\
\text { experiência? }\end{array}$ & 0,495 & 0,693 & * & $*$ & 0,448 \\
\hline
\end{tabular}


Por meio da análise dos componentes principais observou-se a existência de duas dimensões, que juntas responderam por $65,3 \%$ da variância total. A dimensão 1 respondeu pela maior variação nos resultados (eigenvalue $=9,422-55.4 \%$ ), enquanto a dimensão 2 respondeu por um percentual menor de variação no conjunto (eigenvalue $=1,681,10 \%$ ). Por esse motivo (dimensão 1 ter respondido pela maior parte da variação), ambas as matrizes foram analisadas, incluindo a matriz sem rotação e com rotação oblíqua (oblimin). A rotação oblíqua permite que as cargas fatoriais se apresentem corretamente para grupos de variáveis intercorrelacionadas. As comunalidades e as cargas fatoriais podem ser observadas na Tabela 1, que indica, na matriz não-rodada, cargas com valores mais elevados agrupados em uma única dimensão (dimensão 1), indicando que o constructo do TEPT é unidimensional. $\mathrm{Na}$ matriz rodada identificou-se a formação de duas dimensões (dimensão 1 e 2 ). A dimensão 1, de acordo com a Tabela 1, agrupou itens que se relacionam à condição estressante do evento traumático. Já a dimensão 2 agrupou itens sobre as cenas e lembranças da experiência traumática, evidenciando uma possível bidimensionalidade constructo do TEPT.

Com relação à distribuição dos escores gerais da PCL-C com os 103 participantes da amostra, verificou-se uma distribuição não-normal, com assimetria positiva, indicando que houve um grande número de pessoas que responderam a valores de intensidade baixos na escala (Figura 1). Essa distribuição pode ter ocorrido porque muitos dos participantes da amostra não apresentavam TEPT após o rastreamento feito por meio da escala PCL-C. O teste de normalidade (Kolmogorov-Smirnov) apontou que a distribuição difere significativamente de uma distribuição normal $(p<0,001)$. Analisando a distribuição da escala separada em grupos (com diagnóstico provável e sem diagnóstico de TEPT), identificou-se que a distribuição apenas apresentou uma assimetria positiva no grupo de participantes sem diagnóstico provável de TEPT $(n=76)$, pois muitos sujeitos apresentaram escores próximos de zero. Entre os participantes com diagnóstico provável $(n=27)$, mesmo com poucos participantes, a distribuição foi considerada normal $(p=0,069)$. A confiabilidade da escala foi avaliada por meio da consistência interna, apresentando um alfa de Cronbach de 0,94.

Para fazer as análises da curva ROC consideraram-se os participantes com "diagnóstico provável" ( $\mathrm{n}=27)$ e "sem diagnóstico" ( $\mathrm{n}=76)$, obtidos a partir das respostas da PCL-C, cujos escores foram computados com base nos critérios diagnósticos indicados no DSM IV (American Psychiatric Association, 1995). A área sob a curva encontrada foi de $0,949 \quad(\mathrm{EP}=0,020 ; \mathrm{p}<0,001 ;$ I.C. $(95 \%)=0,910-$ 0,987), conforme Figura 1.

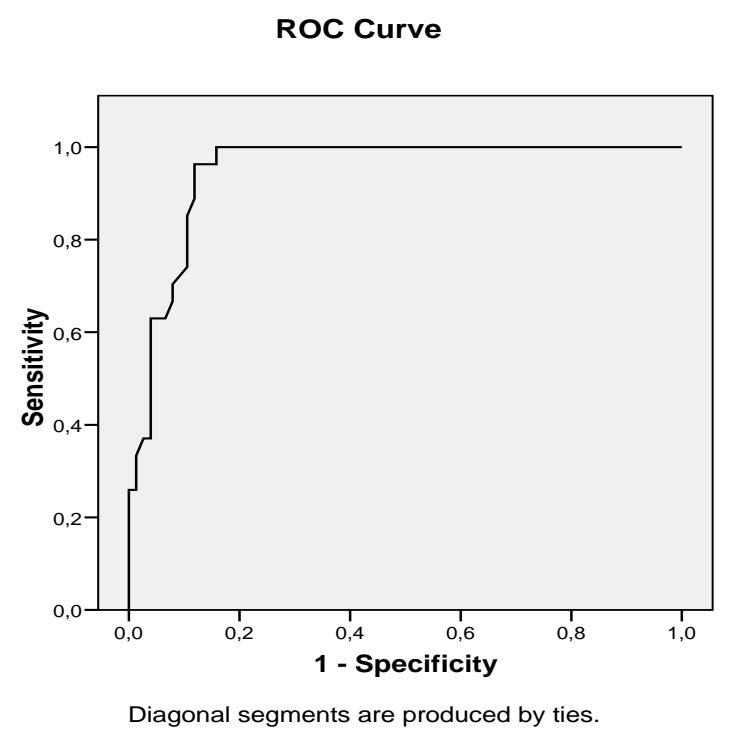

Figura 1. Curva ROC - Receiver Operating Characteristic com os pontos de corte e a capacidade de discriminação da escala PCL-C

Foram obtidos dois possíveis pontos de corte ideais. O ponto de corte que obteve maior

sensibilidade (1) foi 68 pontos, com uma especificidade de 0,842 . O ponto de corte 74 obteve maior 
especificidade em relação ao anterior $(0,882)$, mas perdeu um pouco em sensibilidade $(0,963)$.

\section{Discussão}

Os resultados obtidos nesta pesquisa evidenciam que a PCL-C, de maneira geral, é um instrumento satisfatório para o rastreamento do TEPT. Após enfatizar algumas expressões e a modificação da graduação da escala, observou-se a diminuição das dúvidas dos participantes, melhorando a capacidade de compreensão sobre o que está sendo pesquisado, inclusive como um instrumento de autoaplicação. Ao enfatizar a consigna introdutória "Neste último mês..." seguida da pergunta "Você tem tido...", possibilitou ao leitor uma melhor compreensão de que ele está sendo questionado sobre a reexperiência de um evento passado, no presente. Originalmente o item iniciava como uma frase e encerrava-se como uma pergunta, causando dificuldade de compreensão.

A alteração na intensidade da escala de 1 a 5 para 0 a 10 possibilitou a adequação da consigna nada igual a 0 . Na escala original (Weathers \& cols., 1993) e na versão traduzida (Berger \& cols., 2004), nada é igual a 1, dificultando a compressão do leitor, pois o 1 representa ter, mesmo que de forma mínima, a presença daquele sintoma.

Por meio da análise fatorial exploratória ficou evidenciado que a PCL-C é uma escala unidimensional, pois uma única dimensão respondeu por $55.4 \%$ da variabilidade total da escala. Uma estrutura unidimensional também foi identificada por Maes e cols. (1998b), que encontraram, na matriz sem rotação, um único componente onde todos os sintomas do estresse pós-traumático carregavam, porém, no presente estudo, as cargas são superiores. Apesar de haver uma possível tendência da escala PCL-C ser bidimensional, esta possibilidade foi descartada, pois a segunda dimensão representou apenas $10 \%$ da variabilidade total, com uma porcentagem baixa em relação à tendência unidimensional. Resultado semelhante foi encontrado por Taylor e cols. (1998): uma dimensão respondia por uma variância de 13\% a $38 \%$, enquanto a dimensão com menor variância explicada foi de $8 \%$ a $9 \%$.

Verificou-se comunalidades e cargas fatoriais baixas para os itens 6 e 8 , referentes à dimensão evitação, indicando baixa proporção de variância explicada no conjunto do construto do TEPT. Na pesquisa realizada por Taylor e cols. (1998), também verificaram cargas inferiores a $0,2 \mathrm{em}$ ambas as dimensões no item 8, entre vítimas de acidentes de trânsito. Apesar da carga fatorial e comunalidade baixas identificadas nos itens 6 e 8 deste estudo, a consistência interna da escala foi elevada 0,94. Estudos com amostras maiores acerca da representatividade dos itens 6 e 8 no conjunto do constructo podem evidenciar com maior assertividade quanto à importância destes dois itens.

Com base no resultado da consistência interna, evidenciou-se que a escala PCL-C apresenta boa confiabilidade. Valores elevados acima de 0,82 também foram obtidos em outros estudos realizados com a escala PCL-C, com veteranos de guerras, vítimas de acidentes de trânsito, assalto, abuso sexual, transplante de medula óssea e atividades de mediação em guerra (Andrykowski \& cols., 1998; Blanchard \& cols., 1996; Smith \& cols., 1999; Weathers \& cols., 1993).

Algumas discussões vêm sendo realizadas a respeito do constructo do TEPT e da organização dos critérios contidos no DSM III, IV e IV-TR (Anthony, Lonigan, \& Hecht, 1999; Asmundson \& cols., 2000; Maes \& cols., 1998b; Taylor \& cols., 1998; Witteveen \& cols., 2006). Os atuais critérios diagnósticos do TEPT, estabelecidos por consenso (Asmundson \& cols., 2000; Kristensen, Parente \& Kaszniak, 2006), não têm sido confirmados em estudos empíricos que têm encontrado diferentes dimensões para o constructo do TEPT (Asmundson \& cols., 2000; Blanchard \& cols., 1995; Maes \& cols., 1998a; Maes \& cols., 1998b; Taylor \& cols., 1998; Witteveen \& cols., 2006). Apesar das divergências sobre a estrutura do constructo do TEPT, a dimensionalidade como um todo tem se mostrado satisfatória, de acordo com os índices de confiabilidade nos diferentes estudos realizados (Andrykowski \& cols., 1998; Blanchard \& cols., 1996; Smith \& cols., 1999; Weathers \& cols., 1993).

A escala PCL-C apresentou elevado poder para discriminar quem tem e quem não tem TEPT, conforme área encontrada sob a curva $\operatorname{ROC}(0,949)$. O valor próximo de um indica que o escore está quase sempre certo (verdadeiros positivos e negativos). $\mathrm{O}$ valor encontrado sob a curva ROC sustenta a capacidade discriminativa de forma que, na amostra de 103 participantes, 27 pessoas identificaram a manifestação dos sinais e sintomas do TEPT com valores de intensidade acima de 7 , enquanto 76 pessoas não identificaram tais manifestações, respondendo à escala com intensidades entre 0 e 4 pontos. Para decidir o ponto de corte buscou-se entender as implicações práticas da escala ou o tipo de erro que a mesma incorre. Entende-se ser mais prudente indicar que a pessoa pode ter estresse pós-traumático quando a mesma não tem (falso positivo), do que não indicar quando há suspeita do transtorno, de forma que o ponto de corte indicado é 68 pontos. 
Quanto ao emprego da escala PCL-C como um instrumento apenas de rastreamento do TEPT, consideramos ser uma limitação do estudo. Destaca-se, contudo, que essa limitação não invalida o seu uso para a identificação de possíveis casos, uma vez que para o diagnóstico do TEPT, de acordo com o CID - 10 (Organização Mundial da Saúde, 1993), há fatores predisponentes, como certos traços de personalidade ou antecedentes causais que podem diminuir o limiar para a ocorrência da síndrome ou agravar sua evolução, evidenciando-se a necessidade da realização de investigação sistemática pelo profissional para que a presença do transtorno seja confirmada, apesar do emprego de escalas ou questionários.

As características psicométricas da escala PCLC apresentam resultados satisfatórios para a consistência interna, validade de construto e de critério. Pode-se concluir que a PCL-C é uma escala unidimensional, embora tenha-se evidenciado a importância de estudos adicionais, tanto exploratórios quanto confirmatórios, em amostras maiores.

Quanto à dimensionalidade do construto para o TEPT, esta é uma questão de origem entre as escalas que se baseiam no DSM, o que não invalida a utilização da PCL-C como instrumento principalmente sustentado pelos resultados atingidos na consistência interna. A discussão se refere a como ela está organizada, aspecto que possibilita que novos estudos sejam realizados para conclusões assertivas acerca da dimensionalidade do construto. Ainda com relação ao construto, apesar da consistência interna satisfatória, evidenciou-se a importância da realização de mais estudos exploratórios e confirmatórios quanto aos itens 6 e 8 . Os mesmos apresentaram cargas fatoriais baixas, indicando uma baixa contribuição no conjunto, podendo ser eliminados. Estudos adicionais podem contribuir para uma decisão assertiva quanto à exclusão dos itens indicados.

\section{Referências}

American Psychiatric Association (1987). Diagnostic and statistical manual of mental disorders ( $3^{a}$ ed. rev.). Washington: American Psychiatric Press.

American Psychiatric Association (1995). Manual diagnóstico e estatístico de transtornos mentais: DSM IV ( $4^{\mathrm{a}}$ ed.). Porto Alegre: Artes Médicas.

Andrykowski, M. A., Cordova, M. J., Studts, J. L. \& Miller, T. W. (1998). Posttraumatic stress disorder after treatment for breast cancer: prevalence of diagnosis and use of the PTSD checklist civilian version (PCL-C) as a screening instrument. Journal of Consulting and Clinical Psychology, 66(3), 586-590.
Anthony, J. L., Lonigan, C. J. \& Hecht, S. A. (1999). Dimensionality of posttraumatic stress disorder symptoms in children exposed to disaster: results from confirmatory factor analyses. Journal of abnormal psychology, 108(2), 326-336.

Asmundson, G. J., Frombach, I., McQuaid, J., Pedrelli, P., Lenox, R. \& Stein, M. B. (2000). Dimensionality of posttraumatic stress symptoms: a confirmatory factor analysis of DSM-IV symptom clusters and other symptom models. Behaviour research and therapy, 38(2), 203-214.

Berger, W., Mendlowicz, M. V., Souza, W. F. \& Figueira, I. (2004). Equivalência semântica da versão em português da Post-Traumatic Stress Disorder Checklist - Civilian Version (PCL-C) para rastreamento do transtorno de estresse póstraumático. Revista de psiquiatria do Rio Grande do Sul, 26(2), 167-175.

Blanchard, E. B., Hickling, E. J., Mitnick, N., Taylor, A. E., Loos, W. R. \& Buckley, T. C. (1995). The impact of severity of physical injury and perception of life threat in the development of post-traumatic stress disorder in motor vehicle accident victims. Behaviour research and therapy, 33(5), 529-534.

Blanchard, E. B., Jones-Alexander, J., Buckley, T. C., \& Forneris, C. A. (1996). Psychometric properties of the PTSD Checklist - PCL. Behaviour research and therapy, 34(8), 669-673.

Bremner, J. D. (2004). Does stress damage the brain? Understanding trauma-related disorders from a mind-body perspective. Nova Iorque: W.W. Norton \& Co.

Figueira, I. \& Mendlowicz, M. (2003). Diagnóstico do transtorno de estresse pós-traumático. Revista Brasileira de Psiquiatria, 25(supl. 1), 12-16.

Graeff, F. G. (2003). Bases biológicas do transtorno de estresse pós-traumático. Revista Brasileira de Psiquiatria, 25(supl. 1), 21-24.

Instituto de Pesquisa Econômica e Aplicada (2007). Custos das mortes por causas externas no Brasil. Obtido do World Wide Web <http://www.ipea.gov.br>.

Instituto de Pesquisa Econômica e Aplicada \& Associação Nacional dos Transportes (2003). Impactos sociais e econômicos dos acidentes de trânsito nas algomeracõoes urbanas brasileiras. Brasília.

Kessler, R. C., Sonnega, A., Bromet, E., Hughes, M. \& Nelson, C. B. (1995). Posttraumatic stress disorder in the National Comorbidity Survey. Archives of general psychiatry, 52(12), 1048-1060. 
Kristensen, C. H., Parente, M. A. d. M. P. \& Kaszniak, A. W. (2006). Transtorno de estresse póstramáutico e funções cognitivas. PsicoUSF, 11(1), 17-23.

Maes, M., Delmeire, L., Schotte, C., Janca, A., Creten, T., Mylle, J., Struyf, A., Pison, G. \& Rousseeuw, P. J. (1998a). Epidemiologic and phenomenological aspects of post-traumatic stress disorder: DSM-III-R diagnosis and diagnostic criteria not validated. Psychiatry research, 81(2), 179-193.

Maes, M., Delmeire, L., Schotte, C., Janca, A., Creten, T., Mylle, J., Struyf, A., Pison, G. \& Rousseeuw, P. J. (1998b). The two-factorial symptom structure of post-traumatic stress disorder: depression-avoidance and arousal-anxiety. Psychiatry research, 81(2), 195-210.

Ministério da Saúde (2008). Indicadores e dados básicos: Brasil - 2008. Obtido em 29 de novembro de 2009 do World Wide Web: <http://tabnet.datasus.gov.br/cgi/idb2008/matri z.htm>.

Norman, G. R. \& Streiner, D. L. (2000). Biostatistics: The Bare Essentials. Londres: BC Decker Inc.

Norris, F. H. (1992). Epidemiology of trauma: frequency and impact of different potentially traumatic events on different demographic groups. Journal of Consulting and Clinical Psychology, 60(3), 409-418.

Oliveira, T. M. V. (2001). Escalas de Mensuração de Atitudes: Thurstone, Osgood, Stapel, Likert, Guttman, Alpert. Administração On Line, 2(2), s/n.

Organização Mundial da Saúde (1993). Classificação de transtornos mentais e de comportamento da CID-10: descriçôes clínicas e diretrizes diagnósticas. Porto Alegre: Artmed.

Roso, M. C. (1998). Escalas de avaliação de transtorno do estresse pos-traumático. Revista de psiquiatria clinica, 25(6), 320-325.
Smith, M. Y., Redd, W., DuHamel, K., Vickberg, S. J. \& Ricketts, P. (1999). Validation of the PTSD Checklist-Civilian Version in survivors of bone marrow transplantation. Journal of traumatic stress, 12(3), 485-499.

Taylor, S., Kuch, K., Koch, W. J., Crockett, D. J. \& Passey, G. (1998). The structure of posttraumatic stress symptoms. Journal of abnormal psychology, 107(1), 154-160.

Vasconcellos, E. A. D. (2005). A cidade, o transporte e o trânsito. São Paulo: Pro Livros.

Weathers, F. W., Litz, B. T., Herman, D. S., Huska, J. A. \& Keane, T. M. (1993). The PTSD checklist (PCL): reliability, validity, and diagnostic utility. Obtido em 30 de novembro de 2009 do World Wide

Web: $<$ http://webcache.googleusercontent.com/search ?q=cache:vSpeb15UB8wJ:www.pdhealth.mil/libr ary/downloads/PCL_sychometrics.doc+The+PT $\mathrm{SD}+$ checklist $+(\mathrm{PCL}):+$ reliability, + validity, + and + diagnostic + utility $\& \mathrm{~cd}=1 \& \mathrm{hl}=\mathrm{pt}-$

$\mathrm{BR} \& \mathrm{ct}=\mathrm{clnk} \& \mathrm{gl}=\mathrm{br}>$.

Witteveen, A. B., Van der Ploeg, E., Bramsen, I., Huizink, A. C., Slottje, P., Smid, T. \& Van der Ploeg, H. M. (2006). Dimensionality of the posttraumatic stress response among police officers and fire fighters: an evaluation of two self-report scales. Psychiatry Research, 141(2), 213228.

Yehuda, R. (2002). Post-traumatic stress disorder. The new England Journal of medicine, 346(2), 108-114.

Recebido em outubro de 2009 Reformulado em fevereiro de 2010 Aprovado em abril de 2010 
ANEXO 1 - PCL-C traduzida para o português com alterações

NOME:

DATA:

IDADE: SEXO: $\square \mathrm{M} \square \mathrm{F}$

Instruções: Abaixo, há uma lista de problemas e de queixas que as pessoas às vezes apresentam como uma reação às situações de vida estressantes. Por favor, indique o quanto você foi incomodado por estes problemas neste último mês. Marque: 0 para "Nada"; 1, 2 ou 3 para "Pouco"; 4, 5 ou 6 para "Médio"; 7, 8 ou 9 para "Bastante"; 10 para "Totalmente"

\section{NESTE ÚLTIMO MÊS...}

1. Você tem tido memórias, pensamentos e imagens repetitivos e perturbadores referentes a uma experiência estressante do passado?

2. Você tem tido sonhos repetitivos e perturbadores referentes a uma experiência estressante do passado?

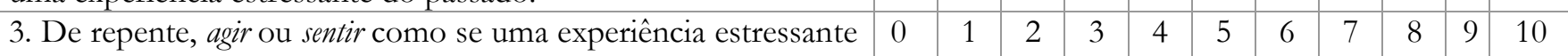
do passado estivesse acontecendo de novo (como se você a estivesse revivendo)?

4. Sentir-se muito chateado ou preocupado quando alguma coisa lembra você de uma experiência estressante do passado?

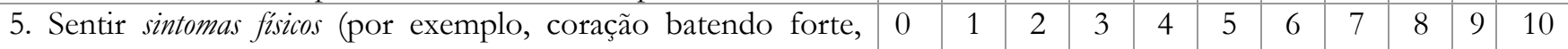
dificuldade de respirar, suores) quando alguma coisa lembra você de uma experiência estressante do passado?

6. Evitar pensar ou falar sobre uma experiência estressante do $00 \mid$\begin{tabular}{l|l|l|l|l|l|l|l|l|l|} 
& 2 & 3 & 4 & 5 & 6 & 7 & 8 & 9 & 10
\end{tabular} passado ou evitar ter sentimentos relacionados a esta experiência?

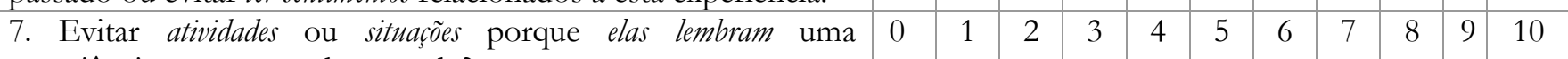
experiência estressante do passado?

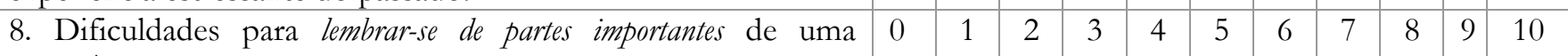
experiência estressante do passado?

\begin{tabular}{|l|l|l|l|l|l|l|l|l|l|l|l|} 
9. Perda de interesse nas atividades de que você antes costumava & 0 & 1 & 2 & 3 & 4 & 5 & 6 & 7 & 8 & 9 & 10
\end{tabular} gostar?

10. Sentir-se distante ou afastado das outras pessoas?

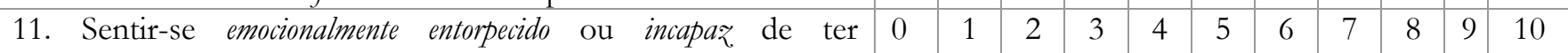
sentimentos amorosos pelas pessoas que lhe são próximas?

\begin{tabular}{l|l|l|l|l|l|l|l|l|l|l|l|} 
12. Sentir-se sem expectativas para o futuro? & 0 & 1 & 2 & 3 & 4 & 5 & 6 & 7 & 8 & 9 & 10
\end{tabular}

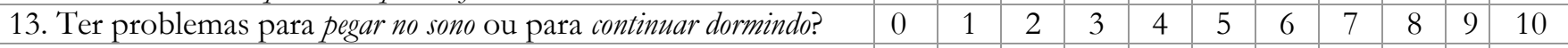

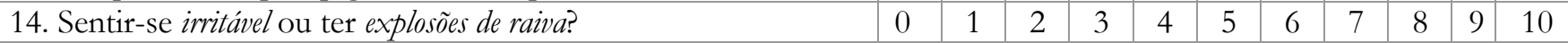

\begin{tabular}{|l|l|l|l|l|l|l|l|l|l|l|l|}
\hline 15. Ter dificuldades para se concentrar? & 0 & 1 & 2 & 3 & 4 & 5 & 6 & 7 & 8 & 9 & 10 \\
\hline
\end{tabular}

\begin{tabular}{|l|l|l|l|l|l|l|l|l|l|l|l|}
\hline 16. Estar "superalerta", vigilante ou "em guarda"? & 0 & 1 & 2 & 3 & 4 & 5 & 6 & 7 & 8 & 9 & 10 \\
\hline 17. Sen i
\end{tabular}

17. Sentir-se tenso ou facilmente sobressaltado? 
Sobre os autores:

Marta Elisa Bringhenti possui mestrado em Saúde Pública pela Universidade Federal de Santa Catarina (2008), aperfeiçoamento em Práticas Psicológicas em Instituições pelo Instituto de Psicologia da Universidade de São Paulo (2004) e graduação em Psicologia pela Universidade do Oeste de Santa Catarina (2002). Trabalha nas áreas da Psicologia e da Saúde Pública nos seguintes temas: Saúde Mental, Epidemiologia, Transtorno do Estresse PósTraumático, Instrumentos de Avaliação Psicológica, Trânsito, Causas Externas, EEG e Neurofeedback.

Caroline Di Bernardi Luft é doutoranda em Psicologia pela UFSC, mestre em Ciências do Movimento Humano UDESC (2007), especialista em Neuropsicologia pela Faculdade Dom Bosco (2004) e graduada em Educação Física (2002). Trabalha com processamento de sinais psicofisiológicos (ex. EEG, HRV) e medidas de fenômenos psicológicos, com ênfase em cognição e instrumentos computadorizados. É responsável pela validade psicométrica dos instrumentos de monitoramento cognitivo desenvolvidos pela Sina-Psi.

Walter Ferreira de Oliveira é graduado em Medicina pela Escola de Medicina e Cirurgia, da Federação das Escolas Federais Isoladas do Estado do Rio de Janeiro, mestrado em Public Health - MPH pela University of Minnesota (1989) e Doctor of Philosophy (Ph.D.), Social and Philosophical Foundations of Education Program também pela University of Minnesota (1994). Atualmente é professor da Universidade Federal de Santa Catarina (UFSC), chefe do Departamento de Saúde Pública. 